\title{
Quadruple Metachronous Malignancy in a Single Patient with Multiple Sclerosis
}

\author{
Marko Buta, Yasuhiro Ito, Ziv Radisavljevic, Zorka Milovanovic, Dragana Lavrnic, Igor Djurisic \\ Merima Oruci, Gordana Pupic, Radan Dzodic
}

\begin{abstract}
Quadruple primary malignancies occur with an incidence of less than $0.1 \%$. Only less than hundred cases have been published until today. The number of multiple malignancies reported is gradually increasing. Here, we present a female patient with a multiple sclerosis and quadruple cancers from different embryological origin. The patient had medullary thyroid carcinoma (stage III-T3, N1a, M0) and multicentric micropapillary carcinomas, two melanomatous lesions, 1.24 and $0.85 \mathrm{~mm}$ thick (Clark II, Breslow II) and breast cancer (T1a, NO, M0). There were no signs of disease recurrence during the 5 years including the exam performed last month. Further genomic studies and closer clinical attention are needed to clarify the relation between secondary malignancies, applied treatments and endogenous and exogenous carcinogens in the process of carcinogenesis in quadruple malignancies.
\end{abstract}

Keywords: Quadruple malignancy, Multiple sclerosis, Carcinogenesis.

How to cite this article: Buta M, Ito $Y$, Radisavljevic $Z$, Milovanovic Z, Lavrnic D, Djurisic I, Oruci M, Pupic G, Dzodic R. Quadruple Metachronous Malignancy in a Single Patient with Multiple Sclerosis. World J Endoc Surg 2012;4(2):79-82.

\section{Source of support: Nil}

Conflict of interest: None declared

\section{INTRODUCTION}

Multiple primary malignancies are two or more malignancies in an individual without any relationship between the tumors. ${ }^{1}$ Multiple malignancies in a single patient are rare but have increased in frequency in recent years. ${ }^{2}$ Multiple primary cancers occurs in 3 to $5 \%$ of malignant tumors and they are frequent in the head and neck, triple tumors occur in only $0.5 \%$, but quadruple tumors in 0.1 to $0.3 \% .{ }^{3,4}$ Quadruple primary malignancies were found in less than hundred cases during the past three decades. ${ }^{5}$ Quadruple cancer, including triple cancers in the head and neck region, was reported. ${ }^{4}$ Quadruple lung cancer in single patient has also been reported ${ }^{6}$ as well as quadruple primary malignancies of the liver, bladder, lung and stomach, all which have been linked to the p53 mutation. ${ }^{1}$ There are similar case reports in the literature describing various combinations of quadruple malignancies affecting almost all organs. ${ }^{7-9}$ We present a case of multiple sclerosis with two different thyroid carcinomas, melanoma and breast cancer.

\section{CASE REPORT}

A 40-year-old woman was diagnosed with multiple sclerosis in 1994 and because of the severe relapsing-remitting course of the disease she was treated with pulsative steroid therapy and short time with azathioprine. Seven years later disease become secondary progressive when patient was treated with mitoxanthrone ( $8 \mathrm{mg} / \mathrm{m}^{2}$ intravenously every 3 months, for 15 months). There were no drug-related serious adverse events or evidence of clinically significant cardiac dysfunction. This therapy abolished the progression of disease. The patient had only moderate spastic paraparesis with gait difficulties. Expanded disability status scale (EDDS) at that time was 5. In family history patient's father had thyroid carcinoma, grandmother had gastric cancer and uncle had pancreatic carcinoma.

In the year 2007, 4 years after discontinuation of mitoxantrone therapy, at the age of 53, patient presented with a cold thyroid, hypoechoic nodule in the right thyroid lobe $42 \mathrm{~mm}$ in diameter and a node of $3.3 \mathrm{~mm}$ in the left lobe. Serum calcitonin value was elevated over $2000 \mathrm{ng} / \mathrm{l}$. The patient underwent total thyroidectomy, central node dissection (pretracheal and paratracheal bilateral) and sentinel lymph node biopsy of both jugular chains. Patient had stage III (T3, N1a, M0) medullary thyroid carcinoma size $45 \mathrm{~mm}$ in the right lobe (calcitonin +, Tg \pm , CEA +, synaptophisin +, NSE +, chromagranin +, bcl2 +) (Fig. 1) and two micropapillary carcinomas in the left lobe,

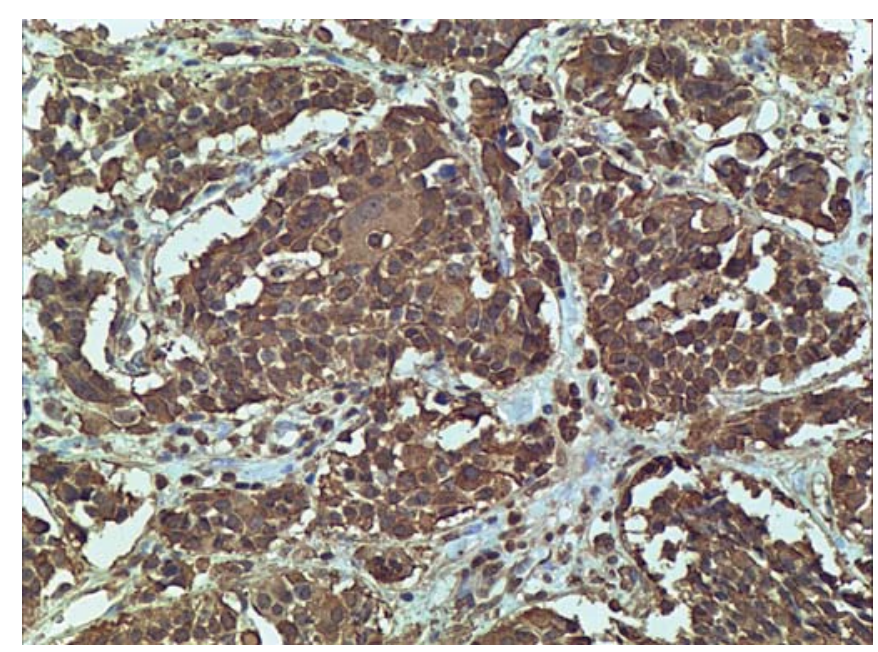

Fig. 1: Medullary thyroid carcinoma anticalcitonin antibody staining of medullary thyroid carcinoma $\mathrm{H}$ and $\mathrm{E} \times 10$ 
size 1.5 and $0.5 \mathrm{~mm}$, medullary thyroid carcinoma metastases in the central neck region (4/12) with negative sentinels bilaterally (0/6 in the left jugular chain and 0/2 in the right jugular chain). Postoperative calcitonin value was 81,7 pg/l, CEA $2.75 \mathrm{ng} / \mathrm{l}$ and whole body scan after 24 hours showed $0.93 \%$ fixation over the central neck region and no fixation over the thorax. Multidisciplinary committee decided a patient should be treated with external $60 \mathrm{~Gy}$ beam irradiation of neck and mediastinum and L-thyroxine suppression therapy.

A year later of the thyroid surgery, in the year 2008, patient complained on enlargement of pigmented skin lesion located over right scapula and other pigmented skin lesion was discovered during the clinical examination in the left lumbar region. Preoperative scintigraphy was performed and one hot node was identified in the right axilla. Both skin tumors were radically excised with more than $10 \mathrm{~mm}$ margins and histopathology revealed 1, $24 \mathrm{~mm}$ thick scapular melanoma-superficial spreading type (Clark II, Breslow II) and $0.85 \mathrm{~mm}$ thick lumbar melanoma-superficial spreading type (Clark II, Breslow II) (Fig. 2) with negative axillary sentinel node.

Fourteen months later, while the perimenopausal patient was on regular follow-up, the breast tumor was discovered on mammography as a stellate lesion $10.8 \times 6.3 \mathrm{~mm}$, BIRADS 5, for which she underwent quadrantectomy with level I and partially level II axillary lymph nodes dissection. Histopathology showed lobular invasive $4 \mathrm{~mm}$ breast carcinoma with LCIS and DCIS (lobular and ductal carcinoma in situ) (HG II, NG II, CK7+, LCA-) (Fig. 3), clear resection margins and negative nodes (0/1). An estrogen and progesterone receptors were strongly positive and human epidermal growth factor receptor-2 overexpression was minimal. Patient underwent $40 \mathrm{~Gy}$ postoperative radiotherapy of the left breast (extended field)

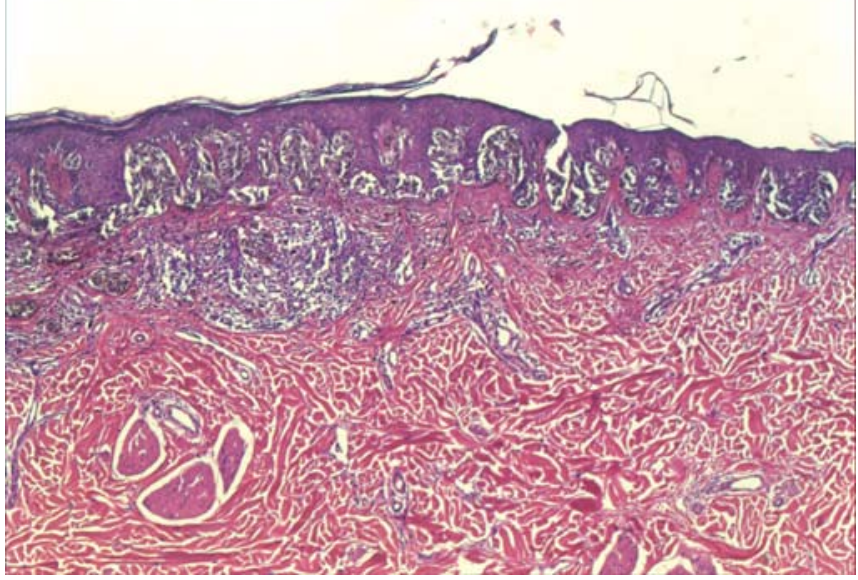

Fig. 2: Skin melanoma invasive cutaneous melanoma $\mathrm{H}$ and $\mathrm{E} \times 10$

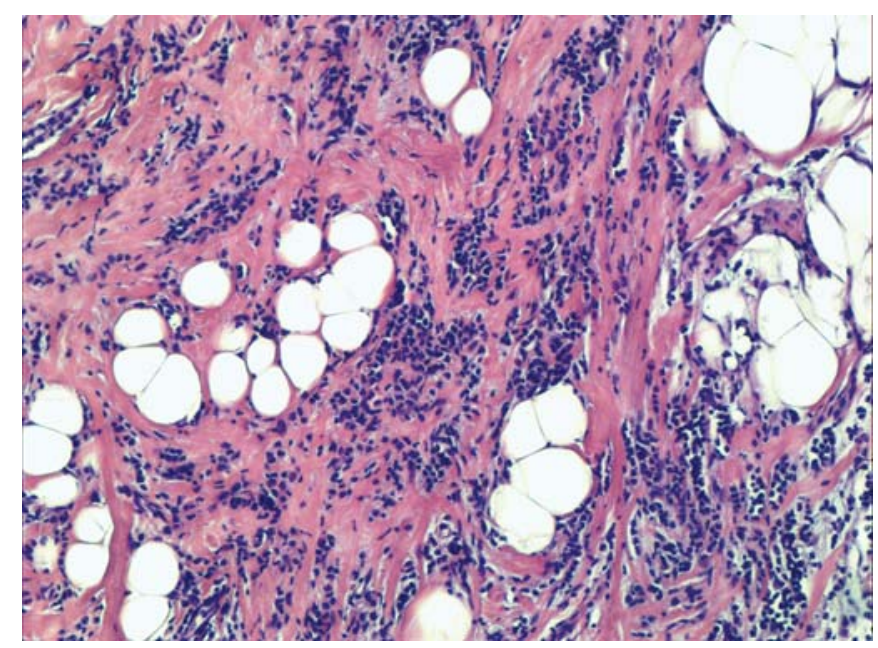

Fig. 3: Breast cancer invasive lobular breast cancer $\mathrm{H}$ and $E \times 10$

considering the fact that she had been previously treated with $60 \mathrm{~Gy}$ in the neck and mediastinum due to thyroid carcinoma. During the course of her treatment, patient became postmenopausal and $20 \mathrm{mg}$ tamoxifen daily was added to her treatment.

Patient is on regular follow-up without signs of recurrence. FDG-PET scan performed in December 2011 was negative, CA 15-3 level was within normal limits and calcitonin level was $83 \mathrm{ng} / \mathrm{l}$ and CEA $5 \mathrm{ng} / \mathrm{l}$. Brain MRI indicated multiple foci of demyelination, without active lesions, and in comparison with the previous MRI findings there were no signs of the disease progression. Also, neurological examination performed in December 2011 did not show any signs of disease progression. She had moderate spastic paraparesis, and EDSS still 5 , as it was 4 years ago.

\section{DISCUSSION}

We report a case of quadruple nonsynchronous malignancy in a single patient with multiple sclerosis where cancers are from different embryological origin. Quadruple malignancy is a rare phenomenon in medicine and most cases of multiple malignancies affect one organ in a female patient. From 1980 to 2011, only 89 cases of primary quadruple malignancy have been reported. ${ }^{5}$ It would be easier to explain multiple carcinomas of same or similar histological origin than tumors originating from different embryological tissues as in our case (melanocytes from neuroectoderm, breast from ectoderm, thyroid gland from endoderm, C cells from neural crest). ${ }^{10}$ There are many possible causes of multiple malignancies, such as genetic mutation and family history, exposure to anticancer chemotherapy, radiotherapy, reduced immunologic response and smoking. Multiple malignances could be in some instances linked to $\mathrm{Li}$ Fraumeni and Lynch syndrome but such an association 
could not be established in our case. Our patient had family history of malignancy on mother's and father's side (father, grandmother and uncle). Patient has not received chemotherapy with alkylating agents which are well known cause of secondary cancers. The patient received mitoxanthrone, a drug extensively used as a diseasemodifying therapy for multiple sclerosis. However, this treatment could be linked to nonmelanocytic skin tumors and increased susceptibility to develop acute promyelocytic leukemia. ${ }^{11-13}$ A high incidence of synchronous or metachronous breast cancer has been reported in patients with malignant thyroid tumor. ${ }^{14}$ The patient was treated with total thyroidectomy, central neck dissection and sentinel lymph node biopsy of both jugular chains. Sentinels were negative bilaterally and prophylactic modified radical neck dissection has not been performed. ${ }^{15}$ The patient was treated postoperatively with 60 Gy external beam radiotherapy (EBRT) of neck and mediastinum but that could not explain development of later breast cancer and melanoma considering short time from exposure to the development of carcinoma.

It is very likely that some genetic alterations were involved in the development of quadruple cancer but genetic testing has not been done yet. The number of multiple malignancies reports is slowly but gradually increasing. ${ }^{7}$ A familial cancers study provided answers and discovery of germ-line mutations of genes during tumorigenesis through loss of heterozygosity $(\mathrm{LOH})$ resulting from somatic deletions or recombinations. ${ }^{16} \mathrm{LOH}$ allowed cloning of $\mathrm{RB}$ gene ${ }^{17}$ and identify the genes that are responsible for familial colon and breast cancers. ${ }^{18-20}$

Epidemiological study have shown an increased incidence of malignancies in patients with multiple sclerosis (MS). ${ }^{21}$ Genetic alterations may affect tumor suppressor genes important in carcinogenesis of quadruple cancers. ${ }^{16}$ RB (retinoblastoma), a tumor-suppressor gene which inhibits cell-cycle progression at the G1/S checkpoint is very important as trigger for cancer proliferation. Rb responds to external growth-factor stimulation by becoming phosphorylated at specific sites, which, in turn, removes the inhibitory influence and allows passage through the checkpoint allowing cancer cell fast proliferation. ${ }^{16}$ FOXO3A gene play important role in development of cancers through the signaling pathway regulating apoptosis NOS/ROCK/FOXO3A. ${ }^{22}$ Cancer growth is supported by the angiogenesis pathay VEGF/PI3K/AKT/NOS/ICAM1 where ICAM-1 is effector protein. ${ }^{23}$ Development of quadruple cancers got extreme robustness by formatting positive loop systems allowing generations of cancer robustness and progression. ${ }^{24,25}$

\section{ABBREVIATIONS}

AKT: Serine/threonine protein kinase (PKB, protein kinase B); LCIS and DCIS: Lobular and ductal carcinoma in situ; EDDS: Expanded disability status scale; FOXO3A: Forkhead box group O; ICAM-1: Intracellular adhesion molecule 1; LOH: Loss of heterozygosity; MS: Multiple sclerosis; NOS: Nitric oxide synthase; PI3K: Phosphoinositol-3-OH kinase; RB (retinoblastoma); mTOR: Mammalian target of rapamycin; VEGF: Vascular endothelial growth factor.

\section{ACKNOWLEDGMENT}

This study was supported by Grant III41031 from the Ministry of Education and Science, Republic of Serbia.

\section{REFERENCES}

1. Yhim HY, Kim HS, Lee NR, Kwak JY, Yim CY, Park HS, Song EK. Quadruple primary malignancies of liver, bladder, lung and stomach in one patient. Tumori 2010;96:787-91.

2. Hu NC, Hsieh SC, Chen TJ, Chang JY. Multiple primary malignancies including colon, stomach, lung, breast and liver cancer: A case report and literature review. Chin Med J (Engl) 2009;122:3091-93.

3. Kurtzke JF. Rating neurologic impairment in multiple sclerosis. An expanded disability status scale (EDSS). Neurology 1983;33: 1444.

4. Németh Z, Czigner J, Iván L, Ujpál M, Barabás J, Szabó G. Quadruple cancer, including triple cancers in the head and neck region. Neoplasma 2002;49:412-14.

5. Kousaka J, Fujii K, Yorozuaya K, Mouri Y, Yoshida M, Nakano S, et al. A case of quadruple primary malignancies including breast, tongue and thyroid cancers and osteosarcoma in a young female without karyotype abnormality. Breast Cancer 2011 May 12. [Epub ahead of print], available at http:// www.springerlink.com.proxy. kobson.nb.rs:2048/content/13406868/?MUD=MPandk=tongue.

6. Goto T, Maeshima A, Oyamada Y, Wakaki M, Hamaguchi R, Kato R. A surgical case of quadruple lung cancer. Ann Thorac Cardiovasc Surg 2010;16:345-50.

7. Angurana SL, Kapoor R, Kumar P, Khosla D, Kumar N, Sharma SC, Patel FD. Quadruple malignancy in a single patient: A case report and comprehensive review of literature. J Cancer Res Ther 2010;6:230-32.

8. Noh SK, Yoon JY, Ryoo UN, Choi CH, Sung CO, Kim TJ, et al. A case report of quadruple cancer in a single patient including the breast, rectum, ovary and endometrium. J Gynecol Oncol 2008;19:265-69.

9. Mitsuhashi M, Yoshimoto M, Matsuyama M, Ito S, Wada S, Kiyota A, et al. A case of quadruple cancer including urinary bladder, oral cavity, stomach and lung. Hinyokika Kiyo 2004;50:429-33.

10. Moore K, Persaud TVN. The branchial and pharyngeal apparatus. The integumentary system. In: The developing human: Clinically oriented embryology, (5th edn). Philadelphia: WB Saunders Company 1993;186-223, 443-50.

11. Hasan SK, Buttari F, Ottone T, Voso MT, Hohaus S, Marasco E. Risk of acute promyelocytic leukemia in multiple sclerosis: Coding variants of DNA repair genes. Neurology 2011;76: 1059-65. 
12. Marriott JJ, Miyasaki JM, Gronseth G, O’Connor PW. Evidence Report: The efficacy and safety of mitoxantrone (Novantrone) in the treatment of multiple sclerosis: Report of the therapeutics and technology assessment subcommittee of the American Academy of Neurology. Neurology 2010;74:1463-70.

13. Mulroy E, Joyce E, Scott J, Melling J, Goggin C, Mahon N, et al. Long-term risk of leukaemia or cardiomyopathy after mitoxantrone therapy for multiple sclerosis. Eur Neurol 2011;67: 45-47.

14. Nio Y, Iguchi C, Itakura M, Toca T, Hashimoto K, Koike M, et al. High incidence of synchronous or metachronous breast cancer in patients with malignant and bening thyroid tumor or tumor-like disorders. Anticancer Research 2009;29:1607-10.

15. Dzodic R, Markovic I, Inic M, Jokic N, Djurisic I, Zegarac, et al. Sentinel lymph node biopsy may be used to support the decision to perform modified radical neck dissection in differentiated thyroid carcinoma. World J Surg 2006;30:841-46.

16. Radisavljevic Z Inactivated tumor suppressor Rb by nitric oxide promotes mitosis in human breast cancer cells. J Cell Biochem 2004;92:511-15.

17. Friend SH, Bernards R, Rogelj S, Weinberg RA, Rapaport JM, Albert DM, Dryja TP. A human DNA segment with properties of the gene that predisposes to retinoblastoma and osteosarcoma. Nature 1986;323:643-46.

18. Groden J, Thliveris A, Samowitz W, Carlson M, Gelbert L, Albertsen $\mathrm{H}$, et al. Identification and characterization of the familial adenomatous polyposis coli gene. Cell 1991;66:589-600.

19. Futreal PA, Liu Q, Shattuck-Eidens D, Cochran C, Harshman K, Tavtigian S, et al. BRCA1 mutations in primary breast and ovarian carcinomas. Science 1994;266:120-22.

20. Narod SA, Salmena L. BRCA1 and BRCA2 mutations and breast cancer. Discov Med 2011;12:445-53.

21. Stich O, Murek C, Rasiah C, Rauer S. Screening for wellcharacterized paraneoplastic antineuronal antibodies in multiple sclerosis. Int J Neurosci 2011;121:477-79.

22. Radisavljevic Z. Nitric oxide suppression triggers apoptosis through the FKHRL1 (FOXO3a)/ROCK kinase pathway in human breast carcinoma cells. Cancer 2003;97:1358-63.

23. Radisavljevic Z, Avraham H, Avraham S. Vascular endothelial growth factor up-regulates ICAM-1 expression via the phosphatidylinositol $3 \mathrm{OH}$-kinase/AKT/Nitric oxide pathway and modulates migration of brain microvascular endothelial cells. J Biol Chem 2000;275:20770-74.

24. Radisavljevic Z. Locus of fragility in robust breast cancer system. J Cell Biochem 2004;92:711-15.

25. Radisavljevic Z. AKT as locus of fragility in robust cancer system. J Cell Biochem 2008;104:2071-77.

\section{ABOUT THE AUTHORS}

\section{Marko Buta (Corresponding Author)}

Surgical Oncology Clinic, Institute for Oncology and Radiology of Serbia, Belgrade, Serbia, e-mail: markobuta@gmail.com

\section{Yasuhiro Ito}

Department of Surgery, Kuma Hospital, Kobe, Japan

\section{Ziv Radisavljevic}

Professor, Department of Cancer Research, Brigham and Women's Hospital, Harvard Medical School, Boston, USA

\section{Zorka Milovanovic}

Department of Pathology, Institute for Oncology and Radiology of Serbia, Belgrade, Serbia

\section{Dragana Lavrnic}

Professor, Department of Neurology, Institute of Neurology of the Clinical Center of Serbia, School of Medicine, University of Belgrade Serbia

\section{Igor Djurisic}

Surgical Oncology Clinic, Institute for Oncology and Radiology of Serbia, Belgrade, Serbia

\section{Merima Oruci}

Surgical Oncology Clinic, Institute for Oncology and Radiology of Serbia, Belgrade, Serbia

\section{Gordana Pupic}

Department of Pathology, Institute for Oncology and Radiology of Serbia, Belgrade, Serbia

\section{Radan Dzodic}

Professor, Department of Surgery, Institute for Oncology and Radiology of Serbia, School of Medicine, University of Belgrade Serbia 